\title{
Allergy to chlorpromazine and valproic acid following carbamazepine hypersensitivity in a patient with an HLA-B*460I allele
}

This article was published in the following Dove Press journal:

Neuropsychiatric Disease and Treatment

\author{
Kumi Sakurada $1, *$ \\ Takeshi Kozaru2,* \\ Kazuo Yamada' \\ Masashi Nibuya' \\ Kiyoshi Nagata ${ }^{3}$ \\ Eiji Suzuki'
}

'Department of Psychiatry,

${ }^{2}$ Department of Dermatology,

${ }^{3}$ Department of Environmental

and Health Science, Tohoku Medical

and Pharmaceutical University,

Sendai, Miyagi, Japan

*These authors contributed equally to this work
Correspondence: Masashi Nibuya Department of Psychiatry, Tohoku Medical and Pharmaceutical University, I-I2-I Fukumuro, Miyagino, Sendai, Miyagi, 983-85I2, Japan

Tel $+8|222344| 8 \mid$

Fax +8I 222752013

Email nibrin@hosp.tohoku-mpu.ac.jp

\begin{abstract}
A 73-year-old man, exhibiting psychomotor excitement after traumatic brain injury, developed allergic cutaneous eruptions and hepatic inflammation that did not resolve after the cessation of carbamazepine (CBZ). Fusing maculopapular erythema was observed in the face, neck, presternal region, and bilaterally in the forearms and feet. A drug-induced lymphocyte stimulation test revealed hypersensitivity to chlorpromazine (CPZ) and valproic acid (VPA), as well as to CBZ. The allergic reaction with eosinophilia to CPZ and VPA was suspected to have emerged following CBZ hypersensitivity, since previous treatment with CPZ and VPA prior to the introduction of CBZ had not been associated with adverse reactions earlier in the course of treatment. Recent studies have indicated linkages between severe CBZ hypersensitivity but not mild CBZ hypersensitivity - and specific leukocyte antigens, HLA-B*1502 and HLA-A*3101, in Asian and European populations. The present case exhibited the HLA-B*4601 allele, which is associated with a high relative risk for the development of CBZ-induced maculopapular eruptions in Japanese and Han Chinese populations. Although cross-hypersensitivity among aromatic compounds, including $\mathrm{CBZ}$ and $\mathrm{CPZ}$, is well-established, data regarding CBZ allergy-associated hypersensitivity to VPA are limited. In the present case, a cutaneous allergy to mianserin (a tetracyclic antidepressant) was also observed later in the course of treatment, suggesting additional cross-reactivity exists among aromatic psychotropic drugs. Thus, the association between the HLA-B*4601 allele and allergic reactions to VPA, aromatic psychotropic drugs, and CBZ should be further examined in future studies.
\end{abstract}

Keywords: human leukocyte antigen, pharmacogenomics, maculopapular erythema, carbamazepine, chlorpromazine, valproic acid

\section{Introduction}

Carbamazepine (CBZ) is widely used in the field of psychiatry to treat epilepsy, the manic state of bipolar disorder, and psychomotor excitement in schizophrenia. CBZ has been reported to cause a wide spectrum of cutaneous adverse drug reactions in $2.9 \%$ of Japanese patients; ${ }^{1}$ the highest rate of allergic rash caused by CBZ treatment has been reported as $10 \% .{ }^{2}$ However, severe adverse drug reactions, including Stevens-Johnson syndrome (SJS) and toxic epidermal necrolysis (TEN), are rare. Findings from recent pharmacogenomic studies have revealed associations between CBZ-induced severe cutaneous drug allergy, including SJS and TEN, and some human leukocyte antigen (HLA) alleles within several ethnic populations in the European ${ }^{3}$ and Asian regions. ${ }^{4,5}$ In Taiwan, a decrease in SJS/TEN has been achieved via HLA genotype-screening. ${ }^{6}$ However, data regarding the association between HLA alleles and CBZ-induced mild cutaneous adverse reactions, including maculopapular eruption, are limited. 
Here, we report the case of a patient who showed a cutaneous drug allergy, with symptoms of hepatic inflammation and eosinophilia, to chlorpromazine (CPZ) and valproic acid (VPA), after exhibiting CBZ supersensitization. The patient possessed the HLA-B*4601 allele, which has been shown to increase CBZ-induced mild cutaneous allergy by 4-6-fold in the Japanese population, ${ }^{1}$ and has been frequently observed in patients with maculopapular eruption in the Han Chinese population. $^{7}$

\section{Case}

A 73-year-old man, who presented with psychomotor excitement and paranoid state caused by a traumatic brain injury on Day 0, was admitted to our psychiatric unit on Day 60.
Edematous contusion and subarachnoidal hemorrhage were observed in the bilateral frontal regions, including the inferior orbital surfaces of his brain, and a conservative treatment approach had been chosen in the neurosurgical unit. To treat the symptoms of psychomotor excitement with delusion and aggression, the psychotropic drugs CPZ and VPA were administered, with CBZ added later (Figure 1). Maculopapular cutaneous eruptions and hepatic inflammation without renal failure were observed on Day 99, following 7 weeks of CBZ therapy. Fused eruptions with exfoliations were observed in the face, entire neck, presternal region, bilateral forearms (including the palms), and bilateral feet (including the plantar dorsum on each foot). Despite the prompt cessation of CBZ followed by the initiation of topical corticosteroids

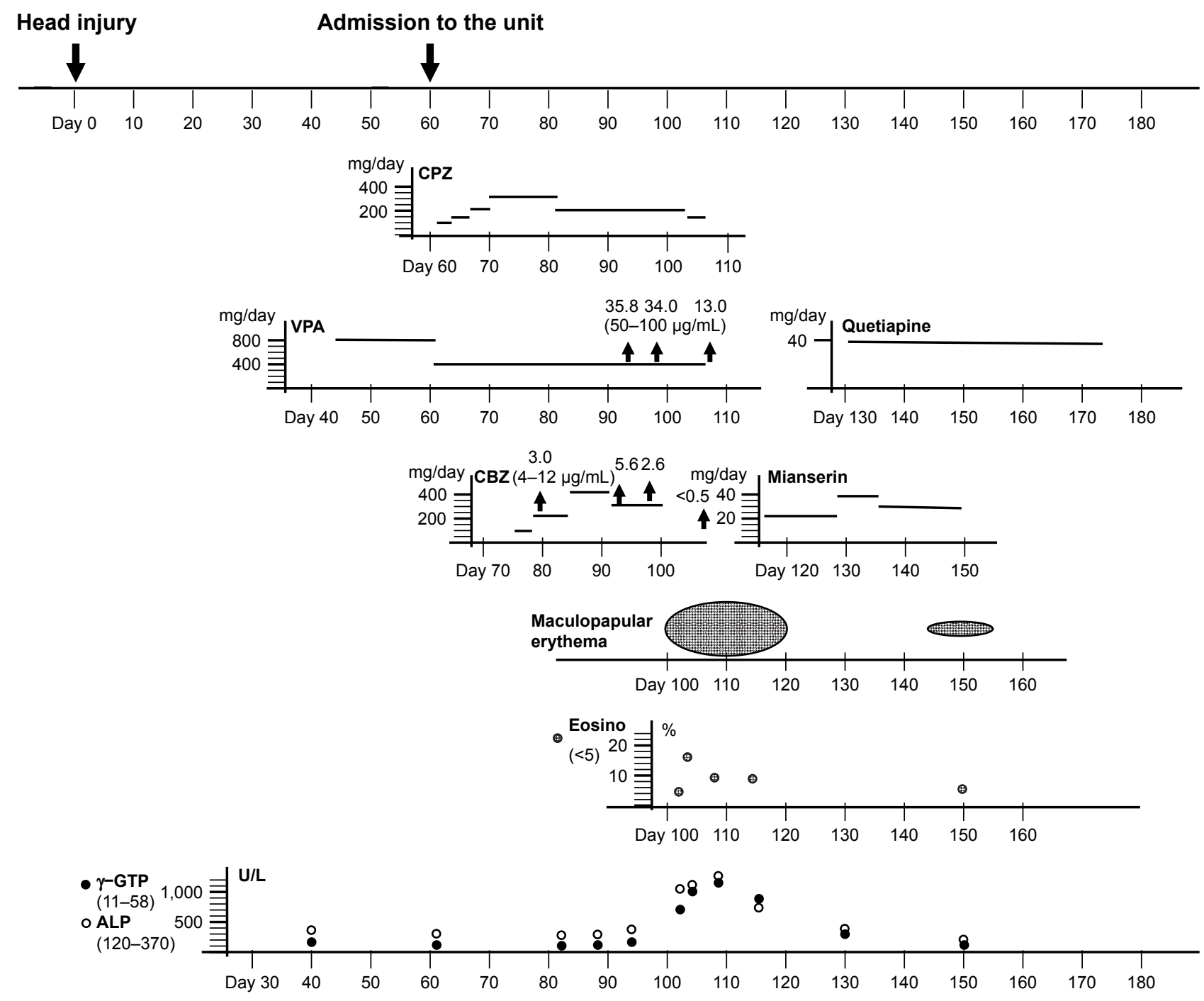

Figure I Schematic drawing of the course of treatment. The day the patient received a brain injury due to a fall down a flight of stairs is set at Day 0 . Administered doses of CBZ, CPZ, VPA, quetiapine, and mianserin are presented in $\mathrm{mg} /$ day. Aside from these drugs, haloperidol $(<3 \mathrm{mg} /$ day) or risperidone ( $<3 \mathrm{mg} /$ day) were used to treat psychomotor excitement with delusional thoughts during the entire course of treatment. Serum concentrations of CBZ and VPA at the indicated time points (arrows) are given in $\mu \mathrm{g} / \mathrm{mL}$. The ratio of eosinophils in the white blood cells is presented as a percentage, and the levels of $\gamma$-GTP and ALP are presented in U/L. The normal ranges for each parameter and the therapeutic effective serum concentrations of the drugs are indicated in parentheses.

Abbreviations: CBZ, carbamazepine; CPZ, chlorpromazine; VPA, valproic acid; $\gamma$-GTP, gamma-glutamyl transpeptidase; ALP, alkaline phosphatase. 
and oral antihistaminergic drugs, the edematous eruptions in the upper and lower limbs, as well as the hepatic inflammation, worsened over the following 10 days. These symptoms began to improve after the cessation of CPZ and VPA. At the peak of the cutaneous eruptions, the following results were obtained from peripheral blood on Day 108: aspartate aminotransferase $($ AST $)=53(7-30) \mathrm{U} / \mathrm{L}$, alanine aminotransferase $(A L T)=215(4-30) \mathrm{U} / \mathrm{L}$, lactate dehydrogenase $(\mathrm{LDH})=264(106-220) \mathrm{U} / \mathrm{L}$, gamma-glutamyl transpeptidase $(\gamma-\mathrm{GTP})=1,126(11-58) \mathrm{U} / \mathrm{L}$, alkaline phosphatase $($ ALP $)=1,200(120-370) \mathrm{U} / \mathrm{L}, \mathrm{C}$-reactive protein=2.22 $(0-0.18) \mathrm{mg} / \mathrm{dL}$, creatinine $=0.90(0.6-1.0) \mathrm{mg} / \mathrm{dL}$, blood urea nitrogen $=17(8-20) \mathrm{mg} / \mathrm{dL}$, white blood cell count $=8,600$ $(4,000-8,000)$ cells $/ \mathrm{mL}$, and eosinophils $=10 \%(0-5 \%)$. During the course of the treatment, fevers (body temperature $>37.5^{\circ} \mathrm{C}$ ) and lymphadenopathy were not observed. The condition was diagnosed as maculopapular erythema because of the lack of symptoms of high fever, which is a criterion for the diagnosis of drug-induced hypersensitivity syndrome. After his recovery from the allergic reaction to $\mathrm{CBZ}, \mathrm{CPZ}$, and VPA, the patient again exhibited milder cutaneous eruptions, possibly induced by mianserin (Figure 1). The eruptions successfully subsided after the cessation of mianserin administration. He reported no previous history of drug or food allergies.

Lymphocyte stimulation test on Day 136 revealed positive reactions to $\mathrm{CBZ}$ (stimulation index $[\mathrm{SI}]=212 \%$ ), $\mathrm{CPZ}$ ( $\mathrm{SI}=245 \%)$, and VPA (SI=383\%). The following were the genotyping (SRL Inc., Tokyo, Japan) results for this patient: HLA-A* was 2402 (35.62\% allele frequency in Japan $)^{8}$ and 0207 (2.87\%); and HLA-B* was 3501 (8.80\%) and 4601 $(3.44 \%)$. The patient and his son provided both oral and written informed consent for the case details to be published.

\section{Discussion}

After the discovery of the significant link between CBZinduced SJS/TEN and the HLA-B*1502 allele in the Han Chinese population in Taiwan, ${ }^{9}$ associations between CBZ-induced severe hypersensitivity and the HLA alleles were found in South-East Asian (HLA-B*1502), Japanese (HLA-A*3101), and European (HLA-A*3101; reviewed by $\mathrm{Su}$ et $\mathrm{al}^{4}$ ) populations. Other associations between psychotropic drug-induced allergies and the HLA alleles have been revealed, including HLA-B*1502 with phenytoin and lamotrigine $^{10}$ and HLA-A*2402 with lamotrigine. ${ }^{11}$ Data from molecular dynamics simulation studies have suggested a possible direct binding of $\mathrm{CBZ}$ to a neighboring region of the antigen-presenting areas of the HLA proteins, ${ }^{12}$ or a binding of HLA-bound CBZ to T cell receptors. ${ }^{13}$ The strong association between the HLA-B*1502 allele and cutaneous CBZ adverse reactions has been observed only in the severe and lethal cutaneous reactions that manifest as SJS and TEN, but not in mild cutaneous adverse reactions that manifest as hypersensitivity syndrome and maculopapular erythema. ${ }^{14}$ In the present case, a fever up and mucous lesions with pain were not observed; thus, a severe cutaneous adverse drug reaction associated with SJS was not diagnosed.

The susceptibility of HLA allele carriers to mild cutaneous drug allergies requires further study. Thus far, most studies have examined the correlations between patients that exhibit a severe drug allergy (SJS/TEN patients) and CBZ-tolerant patients or normal controls. Using this method, patients who exhibited cutaneous adverse reactions without fever and mucous lesions were excluded from the study, despite their intolerance of CBZ administration. Several studies have reported associations between HLA alleles and mild cutaneous adverse reactions to $\mathrm{CBZ}$. The reported relative risks (RRs) of the four HLA-A and -B alleles of the present case, for developing mild cutaneous adverse drug reactions $(n=20)$ to $C B Z$, are as follows: HLA-A*0207 $(R R=3.11)$, HLA-A*2402 $(R R=1.80), H L A-B * 3501(R R=1.19)$, and HLA-B*4601 $(\mathrm{RR}=4.40) .{ }^{1}$ In this Japanese study, the RR of the HLA-B*4601 allele, with respect to the development of severe cutaneous adverse drug reactions to $\mathrm{CBZ}(\mathrm{n}=10)$, was also as high as $6.24 .^{1}$ The ratio of patients with the HLAB*4601 allele $(11 / 28 ; 39.2 \%)$ who had mild maculopapular eruptions without SJS/TEN in central $\mathrm{China}^{7}$ was higher than the reported frequencies of the allele $(4.1 \%-21.6 \%)$ in several regions in China. ${ }^{15}$ A recent study in Taiwan ${ }^{16}$ did not find an association between CBZ-induced mild cutaneous adverse reactions and the HLA-B*4601 and HLA-A*0207 alleles, despite finding a positive association with the HLA-A*3101 and HLA-B*5101 alleles. Another interesting observation is that carriers of the HLA-B*4601 allele are less likely to develop a severe cutaneous adverse reaction (manifesting as SJS/TEN) to CBZ. ${ }^{17}$ The hypothesis that HLA-B*4601 allele is a protective factor with regard to SJS/TEN is consistent with the present case, where no mucous lesions or high fever were observed. In addition, a recent report, using both case-control study and meta-analysis, revealed a positive relationship between the presence of the HLA-A*24:02 allele and maculopapular eruptions upon administration of aromatic antiepileptic drugs. ${ }^{18}$

Our literature review revealed multiple reports showing cross-sensitization among aromatic compounds, including CBZ, CPZ, and tricyclic antidepressants. ${ }^{19,20}$ In the present case, the initial sensitization is attributable to CBZ; it is 
conceivable that $\mathrm{CPZ}$, a compound with a similar tricyclic structure to $\mathrm{CBZ}$, exaggerated the allergic response. Because CPZ and VPA were used safely early in the course of treatment, we suspect that both drugs consequently induced the allergic reaction following sensitization by CBZ. The allergy to mianserin (a tetracyclic antidepressant) may have also been elicited through cross-reactivity of the aromatic compounds following CBZ sensitization. Although VPA is classified as a non-aromatic antiepileptic drug and has fewer risks for developing allergic reactions, ${ }^{21}$ some reports have suggested cross-reactivity of VPA and aromatic antiepileptic drugs, including $\mathrm{CBZ}$ and lamotrigine. ${ }^{22-24}$ Thus, the current recommendation, where VPA and benzodiazepines are regarded as safe alternatives for patients with allergic reactions to aromatic anticonvulsants, ${ }^{25}$ should be re-examined. The present case is a rare instance of allergic responses to multiple psychotropic drugs. Future studies are required to elucidate the role of the HLA alleles, including HLA-B*4601, HLA-A*2402, and HLA-A*0207, in cross-reactivity of psychotropic drugs during the development of cutaneous adverse reactions.

\section{Disclosure}

The authors report no conflicts of interest in this work.

\section{References}

1. Ikeda H, Takahashi Y, Yamazaki E, et al. HLA class I markers in Japanese patients with carbamazepine-induced cutaneous adverse reactions. Epilepsia. 2010;51(2):297-300.

2. Marson AG, Al-Kharusi AM, Alwaidh M, et al. The SANAD study of effectiveness of carbamazepine, gabapentin, lamotrigine, oxcarbazepine, or topiramate for treatment of partial epilepsy: an unblinded randomised controlled trial. Lancet. 2007;369(9566):1000-1015.

3. McCormack M, Alfirevic A, Bourgeois S, et al. HLA-A*3101 and carbamazepine-induced hypersensitivity reactions in Europeans. $N$ Engl J Med. 2011;364(12):1134-1143.

4. Su SC, Hung SI, Fan WL, Dao RL, Chung WH. Severe cutaneous adverse reactions: the pharmacogenomics from research to clinical implementation. Int J Mol Sci. 2016;17(11):pii:E1890.

5. Ozeki T, Mushiroda T, Yowang A, et al. Genome-wide association study identifies HLA-A*3101 allele as a genetic risk factor for carbamazepineinduced cutaneous adverse drug reactions in Japanese population. Hum Mol Genet. 2011;20(5):1034-1041.

6. Chen P, Lin JJ, Lu CS, et al. Taiwan SJS Consortium. Carbamazepineinduced toxic effects and HLA-B*1502 screening in Taiwan. $N$ Engl J Med. 2011;364(12):1126-1133.

7. Wu XT, Hu FY, An DM, et al. Association between carbamazepineinduced cutaneous adverse drug reactions and the HLA-B*1502 allele among patients in central China. Epilepsy Behav. 2010;19(3):405-408.

Neuropsychiatric Disease and Treatment

\section{Publish your work in this journal}

Neuropsychiatric Disease and Treatment is an international, peerreviewed journal of clinical therapeutics and pharmacology focusing on concise rapid reporting of clinical or pre-clinical studies on a range of neuropsychiatric and neurological disorders. This journal is indexed on PubMed Central, the 'PsycINFO' database and CAS,
8. Nakajima F, Nakamura J, Yokota T. Analysis of HLA haplotypes in Japanese, using high resolution allele typing. MHC. 2001;8:1-32 (in Japanese)

9. Chung WH, Hung SI, Hong HS, et al. Medical genetics: a marker for Stevens-Johnson syndrome. Nature. 2004;428(6982):486.

10. Man CB, Kwan P, Baum L, et al. Association between HLA-B*1502 allele and antiepileptic drug-induced cutaneous reactions in Han Chinese. Epilepsia. 2007;48(5):1015-1018.

11. Moon J, Park HK, Chu K, et al. The HLA-A*2402/Cw*0102 haplotype is associated with lamotrigine-induced maculopapular eruption in the Korean population. Epilepsia. 2015;56(10):e161-e167.

12. Jaruthamsophon K, Tipmanee V, Sangiemchoey A, Sukasem C, Limprasert P. HLA-B*15:21 and carbamazepine-induced StevensJohnson syndrome: pooled-data and in silico analysis. Sci Rep. 2017; $7: 45553$.

13. Zhou P, Zhang S, Wang Y, Yang C, Huang J. Structural modeling of HLA-B*1502/peptide/carbamazepine/T-cell receptor complex architecture: implication for the molecular mechanism of carbamazepineinduced Stevens-Johnson syndrome/toxic epidermal necrolysis. J Biomol Struct Dyn. 2016;34(8):1806-1817.

14. Hung SI, Chung WH, Jee SH, et al. Genetic susceptibility to carbamazepine-induced cutaneous adverse drug reactions. Pharmacogenet Genomics. 2006;16(4):297-306.

15. Middleton D, Menchaca L, Rood H, Komerofsky R. New allele frequency database: http:/www.allelefrequencies.net. Tissue Antigens. 2003; 61(5):403-407.

16. Hsiao YH, Hui RC, Wu T, et al. Genotype-phenotype association between HLA and carbamazepine-induced hypersensitivity reactions: strength and clinical correlations. J Dermatol Sci. 2014;73(2):101-109.

17. Wang Q, Sun S, Xie M, Zhao K, Li X, Zhao Z. Association between the HLA-B alleles and carbamazepine-induced SJS/TEN: a meta-analysis. Epilepsy Res. 2017;135:19-28.

18. Shi YW, Min FL, Zhou D, et al. HLA-A*24:02 as a common risk factor for antiepileptic drug-induced cutaneous adverse reactions. Neurology. 2017;88(23):2183-2191.

19. Ljunggren B, Bojs G. A case of photosensitivity and contact allergy to systemic tricyclic drugs, with unusual features. Contact Dermatitis. 1991;24(4):259-265.

20. Seitz CS, Pfeuffer P, Raith P, Bröcker EB, Trautmann A. Anticonvulsant hypersensitivity syndrome: cross-reactivity with tricyclic antidepressant agents. Ann Allergy Asthma Immunol. 2006;97(5):698-702.

21. Baba M, Karakaş M, Aksungur VL, et al. The anticonvulsant hypersensitivity syndrome. J Eur Acad Dermatol Venereol. 2003;17(4): 399-401.

22. Cogrel O, Beylot-Barry M, Vergier B, et al. Sodium valproate-induced cutaneous pseudolymphoma followed by recurrence with carbamazepine. Br J Dermatol. 2001;144(6):1235-1238.

23. Arévalo-Lorido JC, Carretero-Gómez J, Bureo-Dacal JC, MonteroLeal C, Bureo-Dacal P. Antiepileptic drug hypersensitivity syndrome in a patient treated with valproate. Br J Clin Pharmacol. 2003;55(4): 415-416.

24. Bota RG, Ligasan AP, Najdowski TG, Novac A. Acute hypersensitivity syndrome caused by valproic acid: a review of the literature and a case report. Perm J. 2011;15(2):80-84.

25. Mansur AT, Pekcan Yaşar S, Göktay F. Anticonvulsant hypersensitivity syndrome: clinical and laboratory features. Int J Dermatol. 2008;47(11): 1184-1189.

and is the official journal of The International Neuropsychiatric Association (INA). The manuscript management system is completely online and includes a very quick and fair peer-review system, which is all easy to use. Visit http://www.dovepress.com/testimonials.php to read real quotes from published authors. 